\title{
La réception de la télévision locale. Enquête sur les attentes des téléspectateurs de Vosges Télévision
}

\author{
Céline Ségur \\ Centre de recherche sur les médiations \\ Université de Lorraine \\ Celine.segur@univ-lorraine.fr
}

Les ambitions des dirigeants politiques de la IVe République ont fortement influencé la manière dont s'est installée la télévision en France : le projet d'un média comme " instrument culturel au service de l'unité et de l'égalité entre les hommes » s'est imposé (Lévy, 2007, p. 110). La télévision est une nouvelle technique de communication qui est rapidement investie d'une mission d'éducation morale. Pour cela, au début des années 1950, la priorité est donnée à la couverture de l'ensemble du territoire national, tant au niveau des émetteurs que de l'équipement des foyers. Ainsi, en 1960, 13\% des ménages français possèdent un téléviseur, ce chiffre atteint les $62 \%$ en 1974, pour dépasser les 90\% à partir des années 1990 (il est aujourd'hui de 99\%) (Méadel, 2010, p. 274). Cette politique d'implantation, favorisée par le gouvernement, rencontre une ambition de promotion des territoires et cultures locaux : c'est ainsi que sont mises en place des stations régionales, comme l'a décrit et analysé Benoit Lafon (2012). Entre 1950 et 1954 les quatre premières et pionnières stations régionales Télé Lille, Télé Strasbourg, Télé Marseille et Télé Lyon sont inaugurées; elles se caractérisent par la diffusion de programmes - à caractère expérimental et relevant souvent du bricolage - mettant à l'honneur la vie locale. En 1972, la création de la "troisième chaîne » (aujourd'hui France 3), chaîne des régions, incarne un projet de décentralisation et de régionalisation de la télévision. La naissance des premières chaînes de télévision dites locales, inspirée par une ambition similaire, est rendue possible, à partir de 1982, par la loi sur la décentralisation du 2 mars et par le Plan Câble. Diffusées par le câble, elles sont nombreuses mais ont peu d'audience. Par la suite, la commission nationale de la communication et des libertés (CNCL) délivre les premières autorisations pour des services de télévisions locales hertziennes privées à Télé Toulouse (1988), Télé Lyon Métropole (1988) et 8 Mont Blanc (1989). Aujourd'hui, la proximité autant physique que symbolique de la télévision est incarnée à la fois par les antennes locales de France 3 et par les télévisions locales. Rémy Le Champion (2018, p. 82) indique que le Conseil supérieur de l'audiovisuel recensait en 2014 un total de 42 chaines de télévision locales distribuées par la TNT en métropole, et en conventionne 74 diffusées par voie non hertzienne.

Par une communication située sur le mode de l'interpersonnel plutôt que de la masse, les médias locaux contemporains (presse quotidienne régionale, mais aussi gratuits locaux, radios locales ou constituées en réseau régional - France Bleu -, télévisions locales et antennes locales de France 3) ont ainsi pour ambition et/ou vocation de dynamiser la vie locale, d'impliquer les acteurs locaux afin d'assurer la préservation des identités locales. Ils informent, donnent à entendre les citoyens et sont le lieu de représentations identitaires et territoriales. Mais, peuvent-ils être les vecteurs de démocratie locale? Les chercheurs réunis dans le dossier www.democratie locale.fr (coordonné par Éric Maigret et Laurence Monnoyer-Smith pour la revue Hermès en 
2000) s'accordent pour reconnaître qu'il s'agit d'un mythe, car « la démocratie locale se heurte aux mêmes problèmes que la démocratie nationale, mais elle a de surcroît les siens. Loin d'être le lieu d'une communication parfaite, d'une compréhension réciproque et naturelle, le local présente des spécificités qui le rendent particulièrement peu favorable à la formation d'un véritable espace public » (Maigret, 2000, p. 101). Ainsi, Philippe Le Guern et Pierre Leroux (2000) ont montré que la télévision locale ne représente pas le lieu idéal de la démocratie malgré le mythe du public local participatif. En effet, ils ont observé que les médias locaux ont tendance à constituer un frein à la démocratie locale par la reproduction des discours majoritaires dominants. Une explication est un accès au média qui est plus facile pour les représentants locaux du pouvoir, car ceux-ci possèdent les codes inhérents à la communication médiatique. Une seconde explication est la relation d'interdépendance qui unit journalistes et hommes politiques locaux - un phénomène éprouvé également à l'échelle nationale: "Les représentants locaux du pouvoir n'ont pas à payer un droit d'entrée très élevé dans l'espace public médiatisé. C'est a contrario sur les acteurs les plus atomisés et les moins organisés de l'espace public local (du point de vue de la production d'informations) que s'exerce une véritable censure » (ibid., p. 165).

L'étude dont nous rendons compte dans cet article avait pour objectif d'interroger l'existence d'un rapport particulier au territoire et le rôle que peuvent jouer les médias locaux dans la construction des identités locales, du point de vue des publics. Quelles réceptions ont les téléspectateurs des programmes de télévision locale ? Cet article présente les résultats d'une étude ${ }^{1}$ réalisée en 2016 auprès des publics de la chaîne de télévision locale Vosges Télévision ${ }^{2}$. Notre propos débute par une présentation de la chaîne, son positionnement comme chaîne locale et son inscription territoriale. Puis, nous détaillons les conditions méthodologiques de réalisation de l'enquête. Nous verrons enfin que les attentes des téléspectateurs s'affirment autour de revendications fortes en faveur de l'information, du local et de la proximité ${ }^{3}$.

\section{ViàVosges, ex-Vosges Télévision, une télévision locale de service public}

Les chaînes locales jouent un rôle marginal dans l'industrie télévisuelle nationale: économie fragile, audience faible et productivité minime. En effet, elles sont souvent dépendantes des soutiens financiers des institutions publiques locales et d'un potentiel réduit de revenus publicitaires locaux. Si Médiamétrie annonce un chiffre cumulé de près de 13 millions de téléspectateurs pour la télévision locale entre septembre 2014 et juin 2015 (soit 43\% des téléspectateurs ayant accès par voie hertzienne à au moins une chaîne locale), au quotidien l'institut recense 1,3 millions de téléspectateurs, pour une

\footnotetext{
${ }^{1}$ Nous avons réalisé cette étude pour le Centre de recherches sur les médiations (Crem), en collaboration avec Sylvie Pierre, maitre de conférences en SIC à l'université de Lorraine. Nous avons rédigé en juin 2017 un rapport complet à destination de la chaîne de télévision.

2 Vosges Télévision est devenue viàVosges depuis le 1 octobre 2018, parce qu'elle a intégré le réseau national de chaînes de télévisions locales vià. Sa vocation de chaîne de télévision locale et ses missions de service public n'en ont pas été modifiées. Dans cet article, nous faisons le choix d'utiliser la dénomination actuelle de la chaîne lorsque nous en présentons les caractéristiques, et de conserver son ancien nom pour l'exposé des résultats de l'enquête de manière à en préserver la temporalité.

${ }^{3}$ Nous remercions les évaluateurs de ce texte dont les critiques nous ont amenée à clarifier et mieux situer les résultats d'analyse présentés.
} 
durée moyenne d'écoute de 44 minutes $^{4}$ (source : «Études TV locale septembre 2014juin 2015 », Communiqué de presse Médiamétrie du 15 juillet 2015). Par ailleurs, les chaînes locales représentent «1,5 \% des commandes totales de programmes de la production aidée en France» (Le Champion, 2018, p. 82). Néanmoins, dans certains territoires, la notoriété des chaînes locales est importante ainsi que leur part d'audience relative. C'est le cas pour ViàVosges, dont les dirigeants nous indiquaient que, d'après l'enquête Médiamétrie 2012/13 réalisée dans le département des Vosges, l'audience moyenne quotidienne cumulée de la chaîne est plus élevée que celle de certaines chaînes nationales (publiques et privées).

ViàVosges, ex-Vosges Télévision, ex-Images Plus est une station implantée au niveau du département des Vosges (région Grand-Est), née sur le câble en 1990. En 2009, elle devient une Société d'économie mixte (SEM) financée par le Conseil Général des Vosges, les villes d'Épinal (chef-lieu du département) et de Saint-Dié-des-Vosges (2e ville par le nombre d'habitants) et se voit attribuer une fréquence hertzienne par le Conseil supérieur de l'audiovisuel (CSA). Elle émet sur le canal 31 de la TNT hertzienne, vers " une grande partie du territoire vosgien $»^{5}$. La chaîne revendique fortement son statut de télévision de proximité et son inscription territoriale départementale : "ViàVosges permet donc à une majorité de Vosgiens de recevoir leur télévision de proximité ${ }^{6}$. Elle présente les caractéristiques d'une chaîne de télévision de caractère local (Gadras, Paillart, 2013) : une majorité des programmes a pour objectif la mise en visibilité des acteurs locaux. Cela se fait à travers des programmes d'information (comme « Le journal des territoires »), mais aussi dans le cadre d'une émission quotidienne explicitement dédiée à la valorisation d'«initiatives», qui «incarnent les Vosges» (e.g. "La vie en Vosges»). L'émission culinaire («À feu doux»), elle aussi, met à l'honneur les "personnalités de la région». Les activités associatives font l'objet d'un programme quotidien ad hoc, "Qu'on se le dise », qui conjugue les fonctions d'information, d'utilité sociale et de valorisation. Le siège et les studios de la chaîne sont situés dans la ville d'Épinal, chef lieu du département: cette centralisation se retrouve dans la grille des programmes (l'émission «Films à l'affiche" consiste à présenter la programmation cinématographique des salles d'Épinal) ; elle est déplorée par les habitants des autres villes vosgiennes que nous avons rencontrés. Pour autant, les extraits de reportages mis à l'honneur sur le site internet de la chaîne font apparaître explicitement le nom de chaque ville concernée, comme pour témoigner de la diversité des territoires représentés.

ViàVosges, ou plutôt la collectivité territoriale qui l'édite, contracte un certain nombre d'engagements envers le Conseil supérieur de l'audiovisuel qui lui attribue une fréquence. Le contrat d'objectifs et de moyens précise ainsi les missions de service public confiées à la chaîne, qui portent essentiellement sur trois aspects : l'information, la valorisation et l'appropriation identitaire. Il s'agit en effet pour la chaîne de rendre compte de manière exhaustive et diversifiée des actions réalisées spécifiquement sur le

\footnotetext{
${ }^{4}$ Pour information et rappel, et non à titre de comparaison, la durée d'écoute moyenne quotidienne de la télévision en 2016 était de 3h43 (source: Médiamat annuel 2016, www.mediametrie.fr) ; on sait également que « 8 français sur 10 regardent la télévision chaque jour sur leur téléviseur » en 2015 (source : «Global TV », Communiqué de presse Médiamétrie, 14 octobre 2015).

${ }^{5}$ Il est également possible de recevoir la chaîne par les dispositifs numériques contemporains d'accès à internet et aux programmes de télévision.

${ }^{6}$ Citation extraite du site internet de viàVosges, dans la rubrique "À propos de viàVosges ». Accès : https://www.viavosges.tv/Propos-viavosges.html (consulté le 28/11/18).
} 
territoire vosgien. Il s'agit aussi de promouvoir le département et ses habitants. Enfin, la chaîne doit contribuer au sentiment d'appartenance des habitants. ViàVosges se veut être un outil de citoyenneté : les programmes proposés ont clairement pour vocation d'informer, d'éduquer et d'instruire le public. Il est question ici de donner sens aux évènements et à l'actualité, d'en permettre le décryptage et de favoriser la prise de recul par rapport à une information brute qu'il faut développer et traiter. Il est établi que les projets d'installation de l'audiovisuel public dans des territoires à échelle régionale s'inscrivent dans les paradigmes de la proximité et du local : ils sont porteurs d'une ambition affichée, voire enchantée, qui est la proposition d'un service public de proximité, ainsi que l'expliquent Benoit Lafon (2012) pour le cas des télévisions régionales dans l'Hexagone et Bernard Idelson (2013) pour l'arrivée des stations audiovisuelles outre-mer, à La Réunion particulièrement. ViàVosges affiche une ambition qui correspond tout à fait aux missions de service public qui lui sont confiées ${ }^{7}$.

Au sujet des attentes du public vis-à-vis de l'incarnation des objectifs annoncés par la chaîne, les téléspectateurs que nous avons interrogés semblent n'en reconnaître qu'une partie : à la question "Pourquoi regardez-vous Vosges Télévision?", ils ont répondu massivement en faveur de la fonction informative de la chaîne $(72 \%$ des personnes interrogées ont fait le choix de l'item «Elle m'informe sur l'actualité vosgienne »). En revanche, ils ont été peu nombreux à considérer que la chaîne valorisait leurs activités (voir la figure 1); de même, une part minoritaire des individus interrogés (38\%) a consideré que leur sentiment d'appartenance au département était renforcé par l'écoute de la chaîne (figure 2).

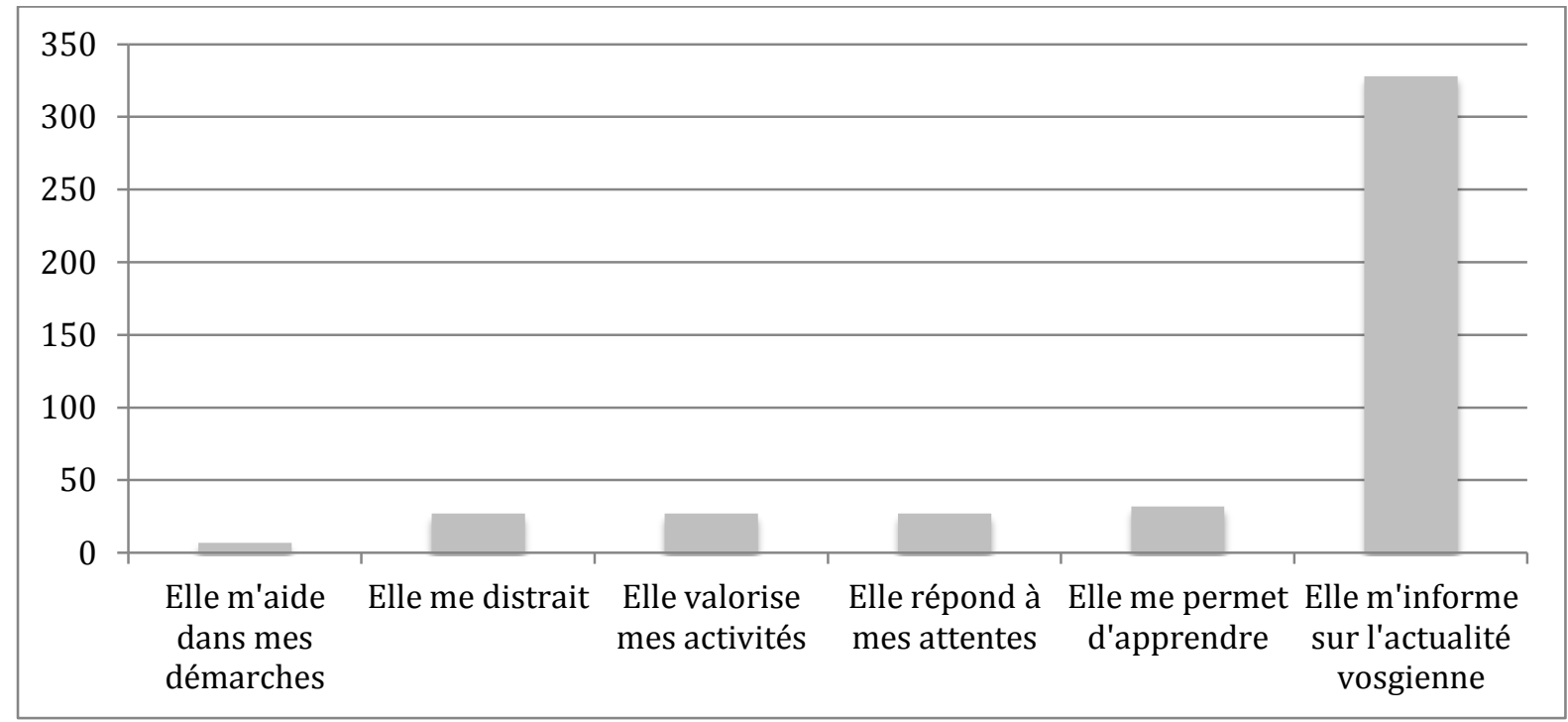

Figure 1. « Pourquoi regardez-vous Vosges Télévision? »

\footnotetext{
${ }^{7}$ « Dès sa création en tant qu'association, viàVosges a été conçu comme un outil de citoyenneté en impliquant le téléspectateur. La chaîne, de par son rôle de service public contribue ainsi à un renforcement de l'identité locale et du sentiment d'appartenance à un territoire ». Citation extraite du site internet de viàVosges, dans la rubrique «À propos de viàVosges ». Accès : https://www.viavosges.tv/Propos-viavosges.html (consulté le 28/11/18).
} 


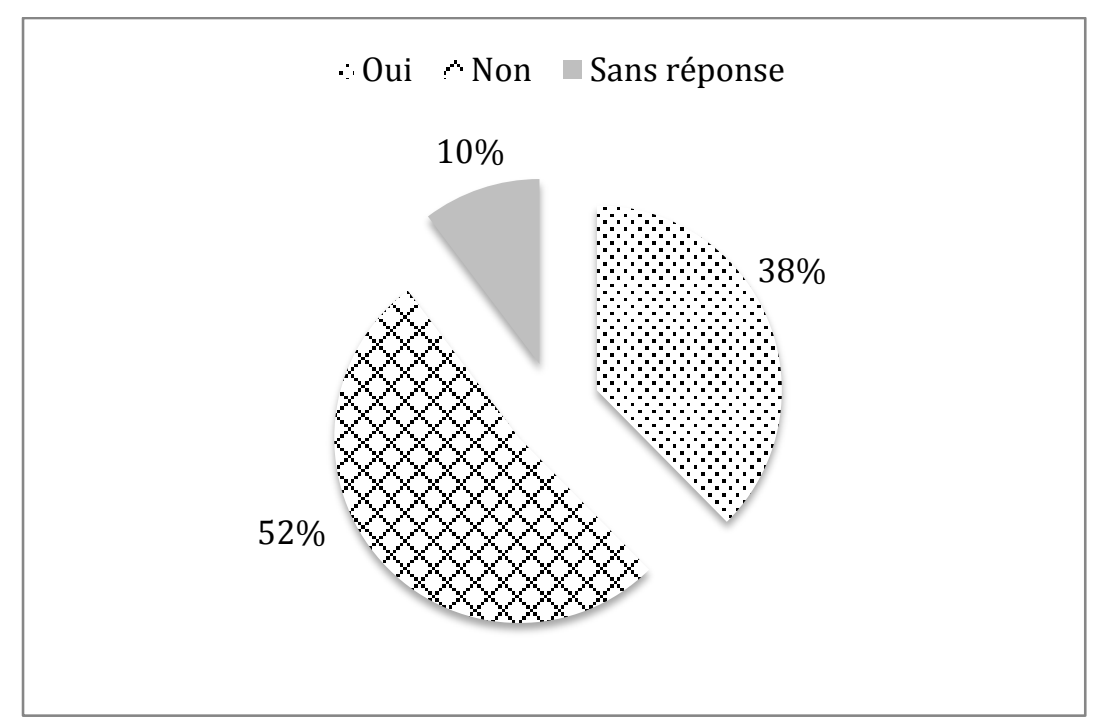

Figure 2. «Ce sentiment d'appartenance au département des Vosges est renforcé par mon écoute de Vosges Télévision ».

\section{Une enquête pour identifier des attentes et des perceptions de téléspectateurs}

En 2014, le Conseil Général des Vosges a commandé, en tant qu'actionnaire principal, un diagnostic global de Vosges Télévision sur "L'analyse des perspectives d'avenir pour Vosges Télévision » (diagnostic remis en février 2014 et présenté au comité d'éthique de la chaîne le 11 mars $2014^{8}$ ). Il en est ressorti la nécessité de disposer d'indicateurs qualitatifs sur les attentes des téléspectateurs. À ce moment-là, la chaîne disposait d'études quantitatives sur son audience, réalisées par l'institut officiel Médiamétrie. L'on sait par exemple qu'environ 140000 individus se déclarent téléspectateurs de Vosges Télévision. Cependant, il est reconnu que les méthodologies quantitatives de mesure de l'audience des programmes télévisuels ne permettent pas de fournir des informations sur les aspects qualitatifs de la relation entre le public et une chaîne de télévision. Ce déficit affecte particulièrement les programmes sur une chaîne de service public (Jost, 2014). C'est pourquoi Vosges Télévision a besoin de connaître la perception du média par ses téléspectateurs ainsi que les attentes du public à l'égard des contenus télévisuels de la chaîne. Ainsi, c'est à la demande de la chaîne que nous avons réalisé, entre 2015 et 2016, une étude localisée auprès de ses publics, dans une double perspective : en tant que chercheurs en sciences de l'information et de la communication, nous avons tenté, d'une part, d'identifier des éléments de connaissance de la perception et des attentes des vosgiens à l'égard de leur télévision locale. D'autre part, notre ambition est de participer à la compréhension des dynamiques qui structurent les rapports contemporains entre les individus et les médias; en particulier, le rapport à la télévision locale comme vecteur de constitution d'une identité territoriale.

L'enquête a débuté par la passation d'un questionnaire auprès des habitants du département des Vosges. Le questionnaire devait pouvoir être renseigné en autonomie, afin d'être diffusé par le site internet de la chaîne et celui du Conseil Général des Vosges. Nous avons opté pour ce mode de passation parce que cela pouvait constituer un mode

${ }^{8}$ Coutinot P., 2014, « Synthèse du diagnostic global de Vosges Télévision », document interne, p. 19. 
d'accès personnalisé aux téléspectateurs de ce territoire ${ }^{9}$. Au regard des objectifs de l'étude, il ne s'agissait pas de dresser un portrait représentatif, dans une approche sociologique, des téléspectateurs de Vosges Télévision, mais de recueillir des traces de sentiment, de représentation, de doléance, d'espoir que pouvaient manifester des individus habitant dans les Vosges, interrogés via un questionnaire au sujet de la chaîne. L'enquête comportait 23 questions fermées (dont quatre destinées à recueillir les renseignements sociodémographiques traditionnels: sexe, âge, catégorie socioprofessionnelle, code postal). Une première partie du questionnaire portait explicitement sur les habitudes d'écoute de Vosges Télévision (fréquence, mode de réception, nature des programmes regardés, etc.). Il s'agissait de recueillir des éléments quantitatifs et qualitatifs sur les pratiques, avec également des questions sur les motivations d'écoute et de non-écoute. Un second moment concernait la perception de Vosges Télévision en particulier, celle des chaînes de télévision locale en général : nous avons proposé aux enquêtés de qualifier leur sentiment et leurs attentes à partir d'un certain nombre d'adjectifs et de fonctions extraites des réflexions récemment conduites autour de la notion de " télévision de qualité » (Jost, 2014). Enfin, il s'agissait d'identifier la place occupée par la chaîne dans la vie des individus, tant d'un point de vue fonctionnel qu'affectif. Sur ce point, les questions ont été formulées à partir des résultats d'enquêtes antérieures sur le rôle de la télévision. Nous nous demandions notamment si l'écoute de Vosges Télévision pouvait être perçue comme un support de sociabilité ( $c f$. Boullier, 1987).

399 individus âgés de 12 à 89 ans ont répondu au questionnaire. Pour des raisons pratiques, le traitement statistique initial brut des données a été réalisé par le Conseil Général des Vosges, qui nous a fourni les résultats en chiffre et en pourcentage pour chaque question, avec un croisement de certaines réponses en fonction des variables sociodémographiques renseignées. Le groupe de répondants se compose d'une légère majorité de femmes (211, pour 181 hommes - 7 individus n'ont pas répondu). Des tranches d'âge (moins de 20 ans, 70-80 ans) sont sous-représentées par rapport à la répartition démographique de la population vosgienne (voir la figure 3$)^{10}$. Au niveau des catégories socioprofessionnelles, les employés et les retraités sont majoritaires dans le groupe (voir la figure 4).

\footnotetext{
${ }^{9}$ Le questionnaire a été diffusé entre le 17/09/15 et le 30/10/15. En plus de la diffusion par les sites internet, une passation a été assurée par les équipes de Vosges Télévision lors d'un salon, ainsi que par nos réseaux personnels.

${ }^{10}$ Des données concernant la répartition démographique de la population vosgienne, issues du recensement 2011, sont disponibles à cette adresse: https://www.insee.fr/fr/statistiques/1379800 (consulté le 20/11/18).
} 


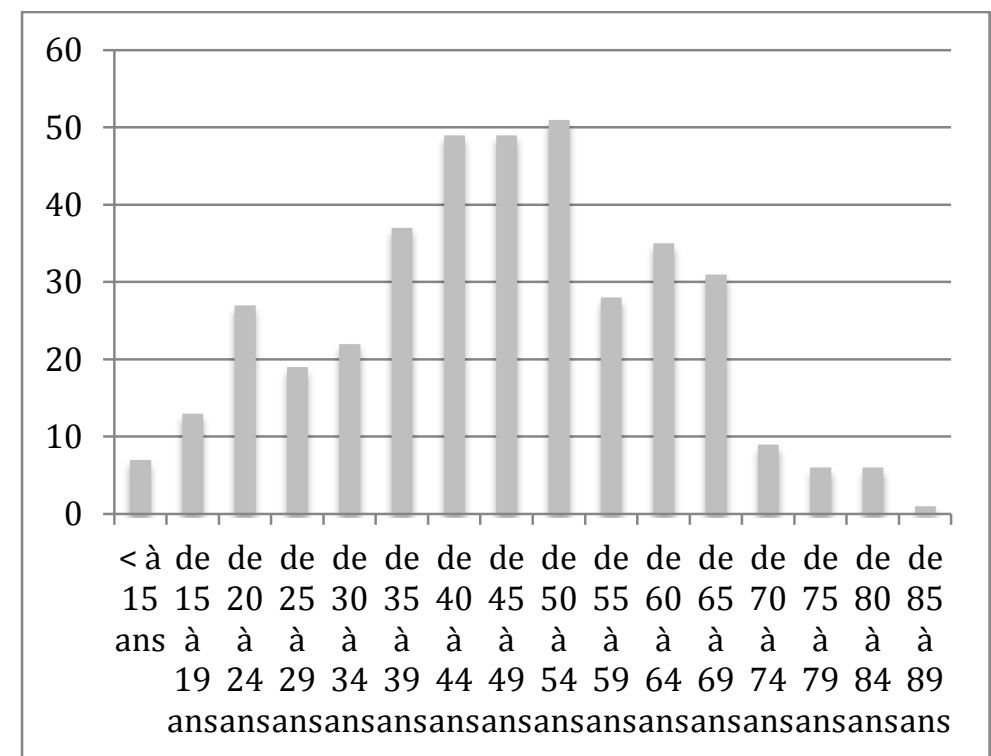

Figure 3. Répartition démographique des enquêtés (en nombre de réponses).

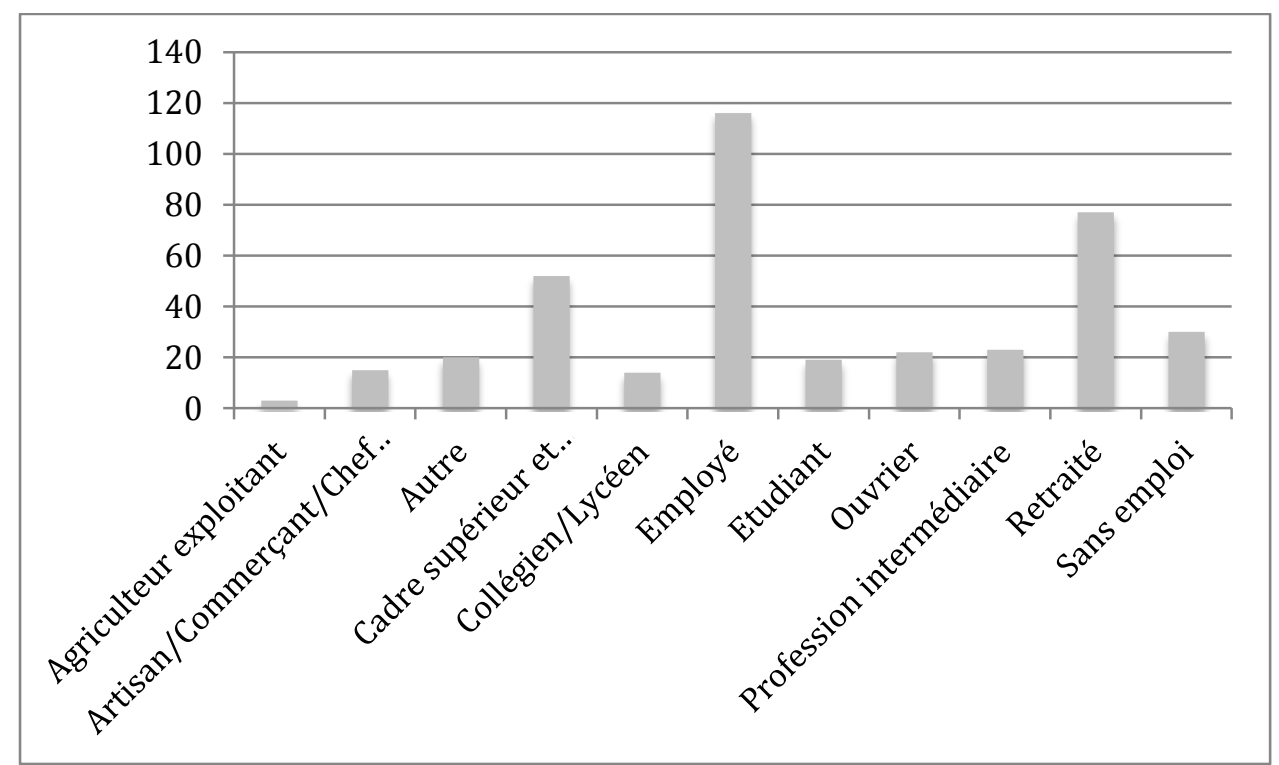

Figure 4. Répartition socioprofessionnelle des enquêtés (en nombre de réponses).

L'enquête s'est poursuivie par l'organisation de trois groupes de discussion. Nous avons choisi cette méthode dans le but de susciter la parole de l'individu tout en lui accordant une certaine liberté d'expression. Il s'agissait d'observer d'éventuelles production et constitution de prises de position sur des sujets, de recueillir des significations partagées et/ou de saisir des désaccords. Nous considérions ainsi que les interactions sociales manifestées dans les discussions pouvaient produire ces prises de position (Duchene, Haegel, 2008). En raison du faible nombre de réponses à notre appel à participation, il n'a pas été possible de réaliser des compositions stratégiques des groupes de discussion ${ }^{11}$. Il est à noter que des liens de sociabilité pré-existaient parmi

${ }^{11}$ Les groupes de discussion ont été organisés et menés par les deux chercheurs qui ont conduit l'étude : Sylvie Pierre et moi-même. Initialement et après lecture des résultats du questionnaire, nous avions prévu de constituer des groupes correspondant à 5 catégories d'individus, définies en fonction de critères d'âge et de rapport à Vosges TV et à la télévision. Mais, une petite annonce diffusée dans le quotidien régional Vosges Matin, à l'antenne et sur le site internet de la chaîne, n'a pas permis de recueillir un nombre de volontaires suffisants pour ce mode opératoire. Nous avons finalement animé trois discussions collectives (environ une heure chacune) auprès : d'un groupe de 6 étudiants de l'ESPE d'Épinal, d'un groupe de 5 
des membres des groupes. Le choix de cette méthode avait pour corolaire la "production d'un discours non formalisé et fluide qui tient de la conversation », une conversation qui articule l'expression d'opinions et celle de normes à l'égard de l'objet d'étude (Lefébure, 2011). En ce sens, les propos tenus par les enquêtés ont été analysés en ce qu'ils traduisent des expériences ordinaires d'un monde vécu, celui du rapport à la télévision, en général, locale, en particulier. Les conversations échangées dans le cadre des groupes de discussion ont, malgré les efforts des chercheurs qui les animaient, porté plus que prévu sur la télévision "en général », que sur la télévision locale de manière spécifique. Ce qui nous amène à penser que la pratique d'écoute de la télévision locale s'insère de manière presque indistincte dans les pratiques de consommation télévisuelle en général, pour ce qui concerne les personnes ici interrogées.

\section{Du désaveu à une réhabilitation numérique}

"Je ne regarde pas trop la télé, sauf depuis que j'ai une tablette et que je peux avoir la télévision par replay»

Le champ des études sur les médias a mis en évidence le contexte symbolique dans lequel s'inscrit toute parole de téléspectateur : le déficit de légitimité dont souffre la télévision dans notre société accompagne régulièrement les prises de position des publics à l'égard de leurs déclarations de pratiques. C'est ce que rappelle Géraldine Poels (2015, p. 13) : " Pratique de masse, d'autant plus incontrôlable et suspecte qu'elle a lieu dans le secret des foyers, l'écoute de la télévision suscite la publication de nombreuses mises en garde destinées à guider les téléspectateurs néophytes, mais aussi à prévenir les éventuelles déviances. Les témoignages de téléspectateurs doivent toujours être interprétés à la lumière de ce contexte symbolique : tout discours sur la télévision est une prise de position, travaillée par le déficit de légitimité croissant de ce média dans les années 60, d'une part, et par le contexte normatif qui détermine les règles de l'écoute profitable, d'autre part». Nous nous référons également aux travaux conduits par Dominique Boulier (1987) sur « les conversations télé » : le chercheur a clairement mis en évidence la posture distanciée adoptée par nombre d'individus lorsqu'il s'agit de rendre compte de sa "culture télé » tant en situation d'entretien avec un chercheur que dans le cadre d'une conversation sur le lieu de travail. Bien que les effets supposés puissants et nocifs de la télévision aient été infirmés (Ségur, 2010), toute discussion publique au sujet de la télévision se révèle porteuse d'un poncif coriace qui se traduit par une mise en récit des pratiques entre désaveu et légitimation.

Les témoignages que nous avons recueillis n'ont pas dérogé à cette "règle »: ils contiennent de nombreux préjugés défavorables à l'égard de la télévision, essentiellement dans un premier moment des discussions. Les discours portent sur la manipulation des médias ("On nous robotise et on nous influence », "C'est vraiment nous prendre pour des idiots, c'est bêtifiant», "C'est pour abrutir le monde », "C'est un instrument de pouvoir, de séduction») et leur toxicité ("J'étais obligée de me désintoxiquer de la télévision", "J'avais l'impression qu'on me prenait ma vie», "Il faudrait une baguette magique pour nous enlever tout ce qu'on nous a mis dans le cerveau»). Les critiques portent principalement sur le rôle de la publicité ( On est bien 
sans la télévision, c'était surtout pour ne plus subir l'agression perpétuelle de la pub», "Ce serait pas mal la télévision, mais sans la pub, agressive tout le temps »), et la manière dont sont construites les informations ("Moi je pense que la télé ça désinforme »). L'éthique de la télévision est remise en cause, elle est un instrument de pouvoir en qui on ne peut pas avoir confiance : "Je pense qu'on est trop guidé par les journalistes, c'est eux qui dominent le monde et ils nous font un lavage de cerveaux, donc moi c'est pour ça que je ne regarde jamais les infos"; "L'info qu'on nous délivre, elle est dénaturée, arrangée ». Nous avons relevé un très fort rejet vis-à-vis des logiques économiques dont TF1 constitue le modèle privilégié, au profit d'une propension à citer des programmes et des chaînes considérés comme plus légitimes (Arte, France 5...).

Face à cela, la légitimation d'une posture de téléspectateur passe par l'affirmation d'un contrôle de la pratique, incarné par la revendication d'un usage important des dispositifs contemporains de replay et des possibilités de visionnage sur d'autres écrans ${ }^{12}$. En effet, les téléspectateurs interrogés affirment leur volonté de ne pas se laisser influencer par la télévision. Les programmes qu'ils regardent ont été choisis ("Je ne regarde pas n'importe quoi [...] Faut surtout cibler»), guidés par la recommandation d'un proche et eux-mêmes se positionnent comme des prescripteurs de programmes considérés comme "intéressants »: "Quand il y a une émission qui démarre et que je sais que ça va intéresser ma fille qui ne regarde jamais la télé, hop un petit texto, du coup, elle va regarder... C'est moi qui fait son programme... et puis on échange en famille »; "De temps en temps mes anciennes collègues, je leur dis tiens, faut voir ça»; "Ça m'arrive de lui téléphoner [à sa mère] en lui disant tiens ce soir regarde ». L'écoute en différé des programmes est particulièrement plébiscitée par les personnes que nous avons rencontrées, cela permet notamment de contourner les publicités ( On choisit son horaire ", "On choisit son programme», "Quand j'ai envie de me mettre devant un truc, c'est pas de la pub, je choisis mon programme et je me le passe »). Surtout, cela permet de contourner la représentation commune de passivité de la pratique télévisuelle. Cela réhabilite la consommation de programmes puisqu'il ne s'agit plus de regarder la télévision, mais de sélectionner un programme sur un écran: "Depuis l'apparition d'internet, internet nous a aidé à devenir actif, à dire je cherche ça, je veux regarder ça, je ne veux pas de pubs »; "Ce qui est positif c'est qu'on peut choisir alors que la tv elle nous rend très passif $»$.

Le questionnaire diffusé auprès de 399 habitants des Vosges a permis de révéler que 38 \% d'entre eux (153) utilisent le site internet de Vosges Télévision pour regarder des programmes en rediffusion; cette pratique étant plutôt masculine en ce qui concerne l'échantillon (84 hommes vs 69 femmes). Le nombre d'individus qui utilisent

\footnotetext{
${ }^{12} \mathrm{Au}$ niveau national, l'écoute de la télévision sur d'autres écrans (smartphone, tablette, ordinateur) tend à se développer, comme la pratique de la catch up tv (écoute en différé). C'est ce qu'indique Julien Rosanvallon, directeur des départements TV et Internet à l'Institut Médiamétrie : en 2017, 8\% de la durée d'écoute quotidienne de programmes télévisuels se faisait sur un écran autre que celui du poste de télévision (soit 19 minutes, un chiffre qui peut atteindre $1 \mathrm{~h} 30$ pour les plus adeptes). La pratique du replay quant à elle a triplé en 3 ans (entre 2014 et 2017), elle concerne à l'heure actuelle 4,6 millions de téléspectateurs, tout écran confondu. Au final, «Live, replay, téléviseur, ordinateur, tablette ou smartphone, loin de se concurrencer, ces écrans et ces modes de consommation des programmes se complètent » (Rosanvallon J., 2017 (25 janv.), Interview : L’année TV 2016 : l'audience augmentée. Accès : http://www.mediametrie.fr/television/communiques/l-annee-tv-2016.php?id=1605. Consulté le 29 mars 2017). Ce qui a conduit Médiamétrie à systématiquement intégrer les audiences des programmes vus en différé dans les résultats d'audience que l'institut publie régulièrement, depuis le 4 janvier 2016.
} 
l'ordinateur pour regarder Vosges Télévision en mode replay est supérieur à ceux qui utilisent l'ordinateur pour regarder la chaîne en direct (153 vs 102). Les catégories socioprofessionnelles de l'échantillon qui sont les plus utilisatrices de Vosges Télévision en mode replay sont les artisans/commerçants/chefs d'entreprise (53\%) ainsi que les cadres supérieurs et professions libérales (52\%). En troisième position se situent les employés (42\%) (figure 5). C'est chez les 30-39 ans de notre groupe d'enquêtés que l'usage de la télévision de rattrapage est le plus important; et, si cet usage décroit avec l'âge passé 40 ans, il demeure relativement important (figure 6). La moitié des usagers du replay (77 sur 153) déclare être prête à regarder davantage la chaîne si celle-ci développait son offre de programmes sur internet. Parmi les non-utilisateurs des programmes en différé sur internet, $26 \%$ des non-usagers déclarent envisager de devenir utilisateurs si la chaîne développait son offre (59 sur 228). Nous pouvons retrouver dans ces résultats la manifestation d'une posture téléspectatorielle active, curieuse d'une consommation à la carte des programmes; elle touche toutes les catégories socio-professionnelles et toutes les tranches d'âge (à l'exception des 70-79 ans) et pourrait, dans certains cas (les collégiens/lycéens/étudiants, les ouvriers, les personnes sans emploi d'une part, les moins de 30 ans d'autre part), faire accroitre de manière relativement importante la proportion d'individus usagers de la télévision de rattrapage (figures 5 et 6).

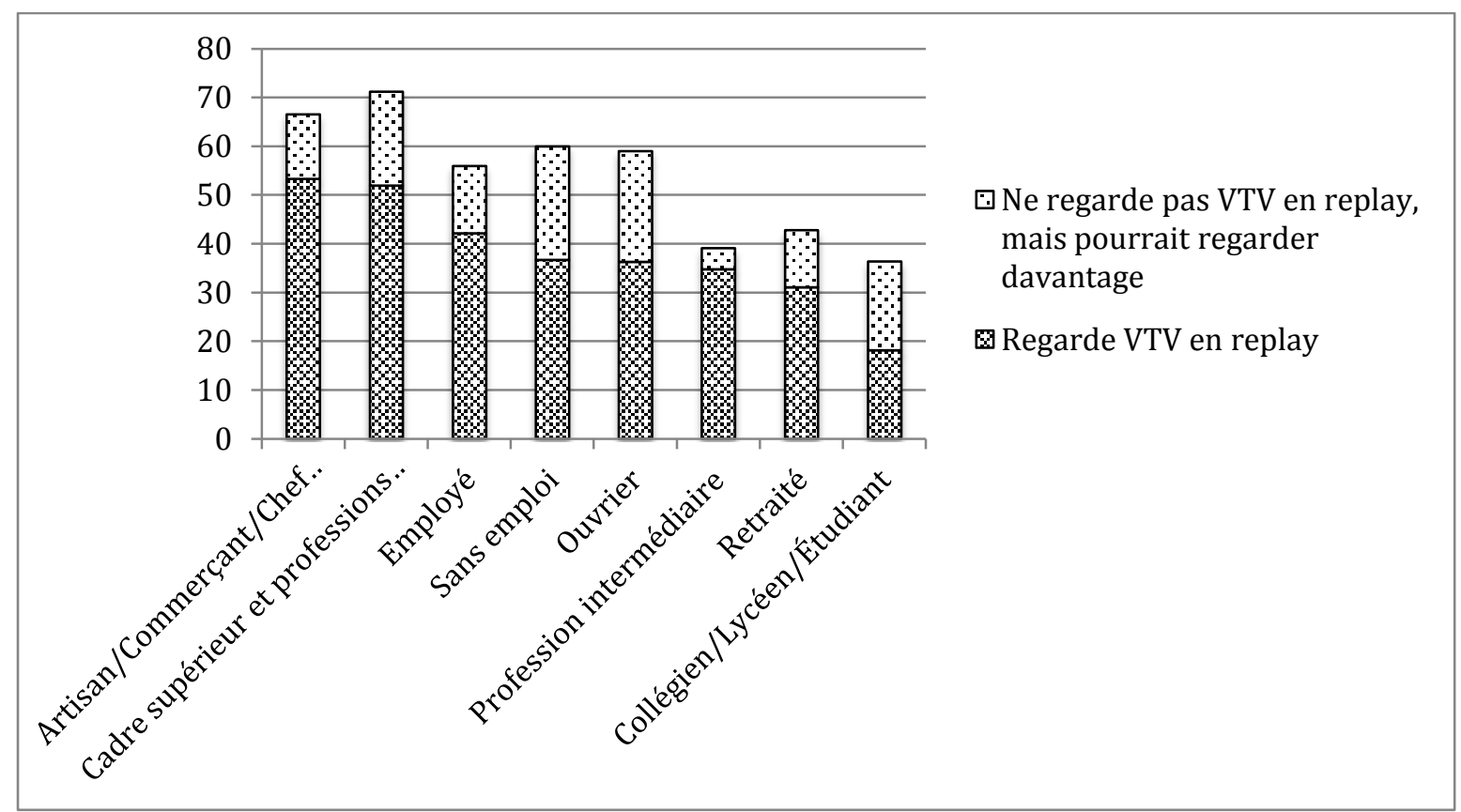

Figure 5. Répartition socio-professionnelle des usagers du replay (\%). 


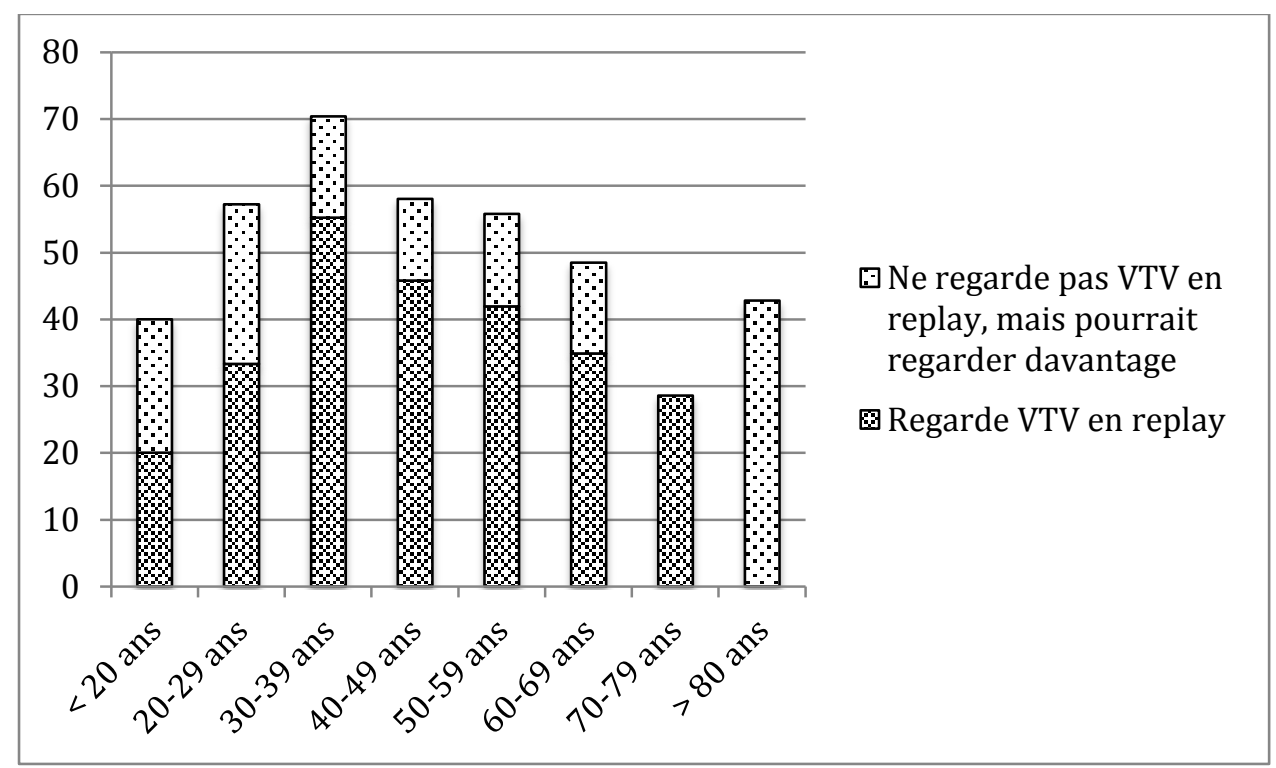

Figure 6. Répartition démographique des usagers du replay (\%).

\section{Pour une télévision actrice de la vie locale}

\section{"Sur Vosges Télévision, on découvre ce qu'il y a à côté de chez nous »}

La chaîne de télévision locale Vosges Télévision souffre d'un manque de visibilité, qui peut nuire à ses résultats d'audience, mais cela n'empêche pas les téléspectateurs d'exprimer de fortes attentes à son égard. Elle semble ne pas être clairement identifiée au sein de l'offre de programmes : "Ce sont des chaînes qui sont loin dans le bouquet de la télévision"; "Je ne sais même pas comment est ce qu'on accède à ces chaines; elles sont toutes dans les chaines qu'on ne connaît même pas tout au bout du bouquet »; ce qui peut expliquer que des personnes ne la regardent pas : "C'est peut-être une méconnaissance des programmes et de ce qui est diffusé ». La plupart ne connaissent pas les programmes diffusés par la chaîne ; plusieurs ne savent pas comment la recevoir : "Ce doit être 142 la chaine, ou quelque chose comme ça [...]. C'est des émissions qui sont répétées assez régulièrement; on peut avoir la même émission plusieurs fois par semaine "; "Vosges Télévision, je sais pas, j'ai jamais vu » " Je ne suis pas certain que mon grand-père sache comment la regarder; par rapport à ce problème de diffusion c'est pas clair pour tout le monde et peut-être que les personnes qui pourraient être intéressées ne sont pas forcément au courant».

Néanmoins, au sujet précisément des télévisions locales, les discours ont été nombreux, et optimistes ${ }^{13}$. En effet, plusieurs personnes ont exprimé un avis positif à l'égard de la télévision locale et voient en elle un média plus authentique, ancré dans la réalité, tout aussi légitime qu'un média national voire plus crédible : "La télé locale, on a peut être un peu plus d'authenticité, on est un peu plus ancré dans la réalité, une réalité locale»;

\footnotetext{
13 L'optimisme se manifeste particulièrement à travers un usage fréquent du mot « positif » pour qualifier les attentes des individus : il s'agirait de montrer "les choses qui marchent»; "Moi je veux positiver, on nous montre toujours le négatif»; "Des choses positives, des choses bien qui sont arrivées ; des gens qui ont monté une entreprise; des gens à qui il est arrivé quelque chose de sympathique»; "Des émissions qui montrent positivement différents métiers ».
} 
"Quand ça m'arrive de, parce qu'il y a quelque chose qui m'intéresse, c'est pas plus nul qu'une autre télévision, qu'une autre chaine locale » ; "Vu à priori qu'il y a moins d'argent à se faire sur une télé locale, on pourrait se dire que du moins c'est plus de liberté ». Le regard sur la publicité diffère aussi selon que la chaîne soit nationale ou locale. Alors que le rejet est fort d'une manière générale, il est plus nuancé pour une chaîne locale car la publicité est perçue comme un moyen de financer les programmes : "Ils ont de la pub, ça les aide à vivre».

Ainsi la télévision locale semble-t-elle incarner l'espoir d'un média d'information qui pourrait jouer un rôle de médiation locale. Vosges Télévision est clairement identifiée comme un média d'information : 84\% des individus interrogés ont déclaré qu'ils regardent la chaîne parce qu'elle les informe sur l'actualité de leur territoire départemental. De fait, le « Journal » quotidien est le programme qui est le plus regardé (il est cité par 94\% des individus), et ce, le plus régulièrement : 41\% l'ont affirmé, alors que la chaine n'est pas regardée au quotidien pour une majorité de l'échantillon. Plus globalement, 4 des 5 programmes identifiés comme appartenant au genre " information » sont les quatre programmes que les individus déclarent regarder le plus ( "L’Hebdo», «Sur le vif», «La vie en Vosges» et la « Météo»). Cette appétence pour l'information, a fortiori locale, se confirme au moment de l'expression des attentes des individus: 83\% d'entre eux souhaitent voir un maximum d'informations locales à l'écran, quelle que soit leur catégorie socio-professionnelle (voir la figure 7). La retransmission des événements locaux et la valorisation des initiatives locales sont également plébiscitées par une majorité. C'est particulièrement le cas des artisans, commerçants et professions intermédiaires (respectivement $73 \%$ et $78 \%$ ont cette attente, contre une moyenne de 58\%) (voir la figure 8).

Une program. identique à celle d'une chaîne.

Le lieu de l'expression de tous les élus

Le lieu de l'expression des citoyens

Des programmes sur la vie quotidienne

Des informations pratiques (emploi, santé)

La promotion d'œuvres audiovisuelles locales

La valorisation des intiatives locales

La retransmission des événements locaux

Un maximum d'information locale

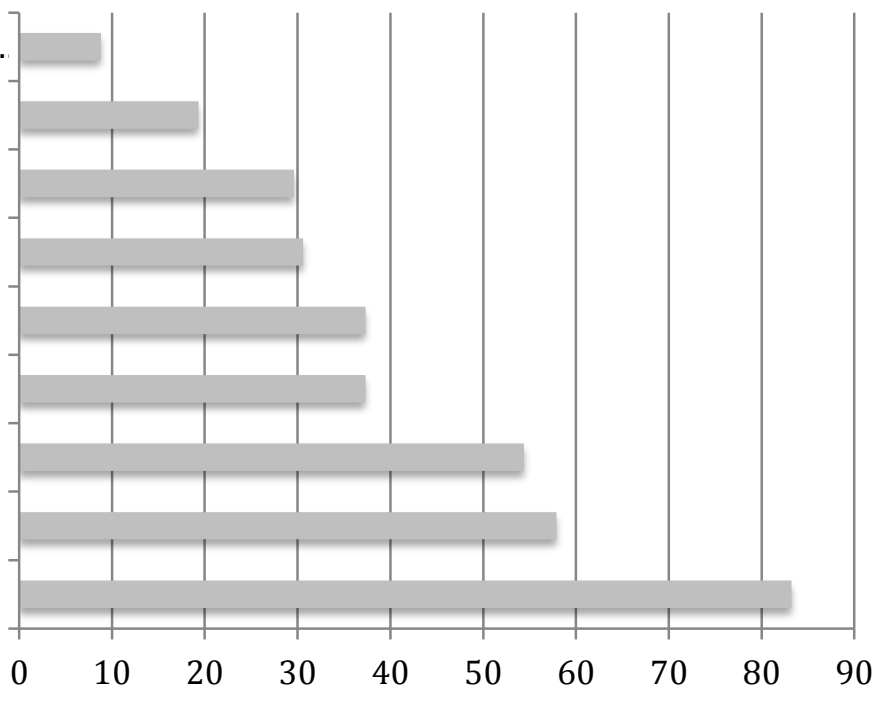

Figure 7. Attentes à l'égard d'une chaîne de télévision locale (\%). 


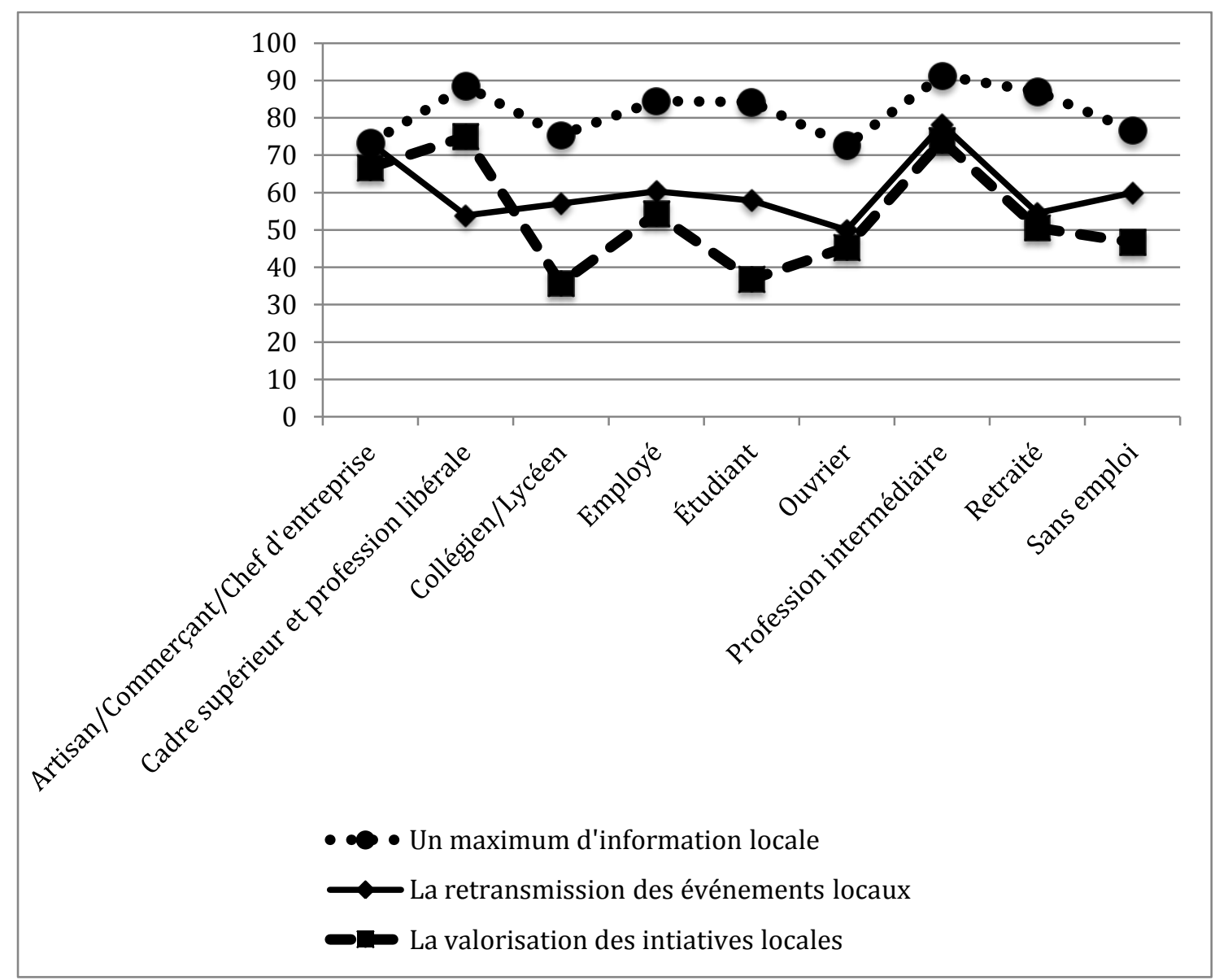

Figure 8. Attentes à l'égard d'une chaîne de télévision locale selon la catégorie socioprofessionnelle (\%).

Au-delà de l'information, les téléspectateurs manifestent une appétence pour la découverte et la connaissance. Ceux-ci souhaitent des contenus approfondis, qui expliquent et leur apprennent quelque chose, "des choses qui nous donnent envie de vivre, de réfléchir»: ainsi cette téléspectatrice explique qu'elle regarde la télévision parce qu'elle est "curieuse», c'est pourquoi elle recherche des programmes qui la nourrisse. Sont ainsi plébiscités " certains reportages, j'aime bien les émissions qui collent à l'actualité et qui l'expliquent, qui font une recherche, une analyse sur ce qui se passe [...] parce qu'on a souvent des émissions où on nous rapporte des faits 5 minutes et puis on ne connaît pas le contexte, ni les tenants et les aboutissants ». Un avis partagé par d'autres: "C'est dans l'air: c'est des sujets très divers... c'est intéressant parce qu'il y a des spécialistes qui nous expliquent ce que nous n'avez pas compris vous-mêmes ».

La fonction informative du média se double d'une fonction sociale de médiation : il s'agit de mettre en valeur les actualités locales. La chaîne a véritablement un rôle à jouer en tant que média de proximité, acteur de la vie locale : «Ce qui est intéressant dans ces télés par rapport aux chaînes nationales c'est vraiment la vie locale, on découvre ce qu'il y a à côté de chez nous ; ça permet aussi de découvrir tout ce qui se fait à proximité; ça permet d'avoir une ouverture sur ce qui se fait localement au niveau associatif, manifestations culturelles, petites entreprises qui s'ouvrent, qui se créent, l'artisanat, enfin des tas de choses qu'on peut découvrir ». En effet, au cours des discussions, il a été révélé que le principal vecteur d'identification de la chaîne concerne le souvenir de la présence d'une 
équipe de tournage lors d'une manifestation locale ${ }^{14}$, plutôt qu'une émission ou un animateur qui incarnerait l'identité du média, comme cela peut être davantage le cas pour les grandes chaînes nationales. Le public que nous avons rencontré souhaite que Vosges Télévision soit davantage présente sur le terrain et qu'elle valorise le territoire local. Les personnes ont à cœur la promotion des actions et activités de proximité, du quotidien ou de l'exceptionnel. Elles attendent une télévision qui soit à la fois le témoin et l'actrice de sa vie locale : "Le plus gros reproche que je ferais à la télé locale c'est d'être juste informative. Pour moi, une télé locale c'est une télé qui s'investit dans le patrimoine, pas que dans le patrimoine, dans le développement de la culture justement, il y a du territoire. Et c'est pas juste une télévision qui informe, c'est une télé qui crée du spectacle et de l'action culturelle ». Il s'agirait de promouvoir le territoire et les initiatives locales, tout en impliquant le téléspectateur, ont-ils tous affirmé : "On doit être au cour de l'action ».

En effet, depuis quelque temps, la communication télévisuelle est placée sous le régime de la participation : les publics manifestent leur volonté de ne plus simplement être les destinataires de produits médiatiques, mais de pouvoir réagir et interagir : "La télé peut être un moyen d'expression des citoyens ». Cette tendance se manifeste dans les propos que nous avons recueillis, non pas au sens d'une «culture de la convergence » (Jenkins, 2013) ${ }^{15}$, mais au sens d'une participation encouragée par une proximité territoriale. En effet, "il faut que ce soit quelque chose de vivant, qu'on se sente investit. Je regarde la télé quand c'est quelque chose qui me touche». Participer rime ici avec proximité, et cela passe par l'ouverture de l'antenne aux téléspectateurs. Ce désir semble aller de pair avec la défense d'une posture du téléspectateur actif manifestée par les participants au sujet des conditions de visionnage de la télévision (cf. supra). Spontanément, plusieurs personnes ont exprimé le souhait de s'impliquer en participant aux contenus télévisuels: "Je pourrais passer à la télé pour expliquer mon point de vue "; "Ça me plairait de participer à un jeu; ça me fait penser au jeu des 1000 euros; je trouve que les jeux c'est bien; ça te permet de découvrir la région les gens; le concept de faire aussi jouer la population; les gens deviennent acteurs, ils cessent d'être des veaux qui regardent passer les trains». Enfin, l'utilité sociale d'une meilleure connaissance des images télévisuelles et de leur fabrique - "Décrypter l'envers du décor, comment on fait 》 - est également soulignée de manière consensuelle : «Passer à la télé, ça fait partie pour moi de la démystification de l'image, se dire moi aussi je peux le faire et moi aussi je peux dire ce que j'ai à dire "; "À l'école, je pense que du point de vue de la classe, le fait de dire on peut faire venir la tv, faire participer au projet de classe, ça peut apporter un plus ; ça peut permettre de regarder l'école avec un autre regard ».

Néanmoins, pour certaines des personnes interrogées, le rapport au local ne peut pas passer par le truchement d'un écran de télévision : "J'ai d'autres choses à faire, je préfère aller au concert, au spectacle, à l'exposition plutôt que d'entendre par l'intermédiaire de l'écran ». De même, Vosges Télévision ne semble pas remplir un rôle de renforcement de l'identité locale (Boure, Lefebvre, 2000, p. 274). Si 86\% des personnes

\footnotetext{
${ }^{14}$ Dominique Boullier (2014, p. 76) faisait l'hypothèse d'un plaisir de la représentation à l'écran d'un élément lié à son univers personnel : ceci est ici manifeste.

${ }^{15}$ La nature du questionnaire et des conversations n'ont pas permis de faire émerger la question de la convergence qui tend à faire disparaître la notion de chaîne de télévision, c'est-à-dire si une offre de programmes locaux à la carte serait attendue de manière à être combinée avec les propositions des autres chaînes.
} 
ont déclaré, via le questionnaire, se sentir «vosgiennes », seulement 37\% d'entre elles affirment que «ce sentiment est renforcé par l'écoute de Vosges Télévision » (figures 9 et 10$)$. Les hommes ( $50 \%$ de ceux qui se sont "déclarés » vosgiens) ont davantage que les femmes (40\%) ce sentiment. Au niveau des tranches d'âge, il apparaît nettement que ce sentiment est renforcé chez les plus âgés du groupe (figure 11). En ce qui concerne les catégories socioprofessionnelles (figure 12), les avis sont partagés pour les retraités, sans emploi, artisans/commerçants/chefs d'entreprise et ouvriers, avec une certaine majorité de "oui, mon sentiment d'être vosgien est renforcé par mon écoute de VTV ». En revanche, du côté des cadres supérieurs, professions intermédiaires, collégiens, lycéens et étudiants, le sentiment d'identité territoriale n'est majoritairement pas relié à la chaîne de télévision.

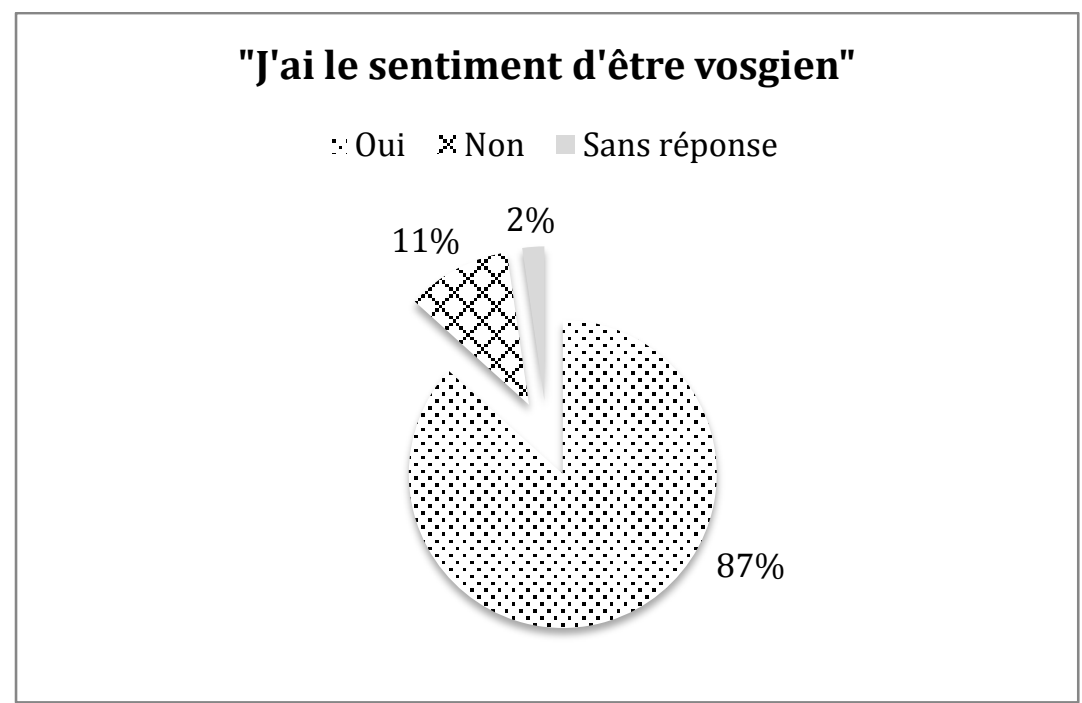

Figure 9. Identité vosgienne.

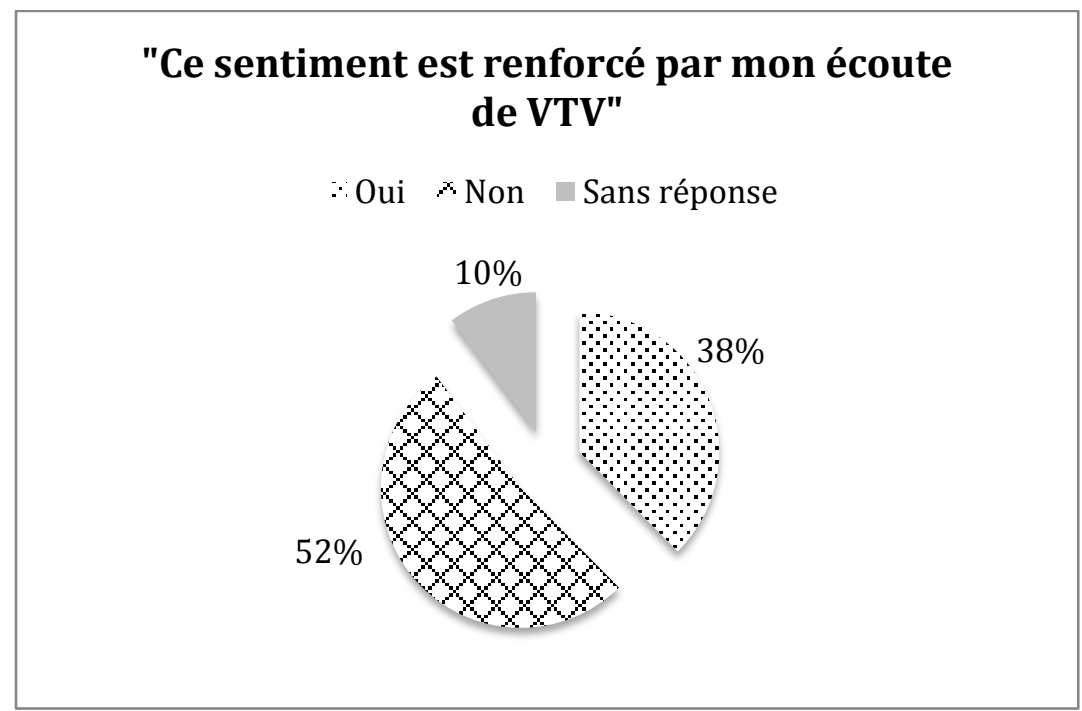

Figure 10. Identité vosgienne et VTV. 


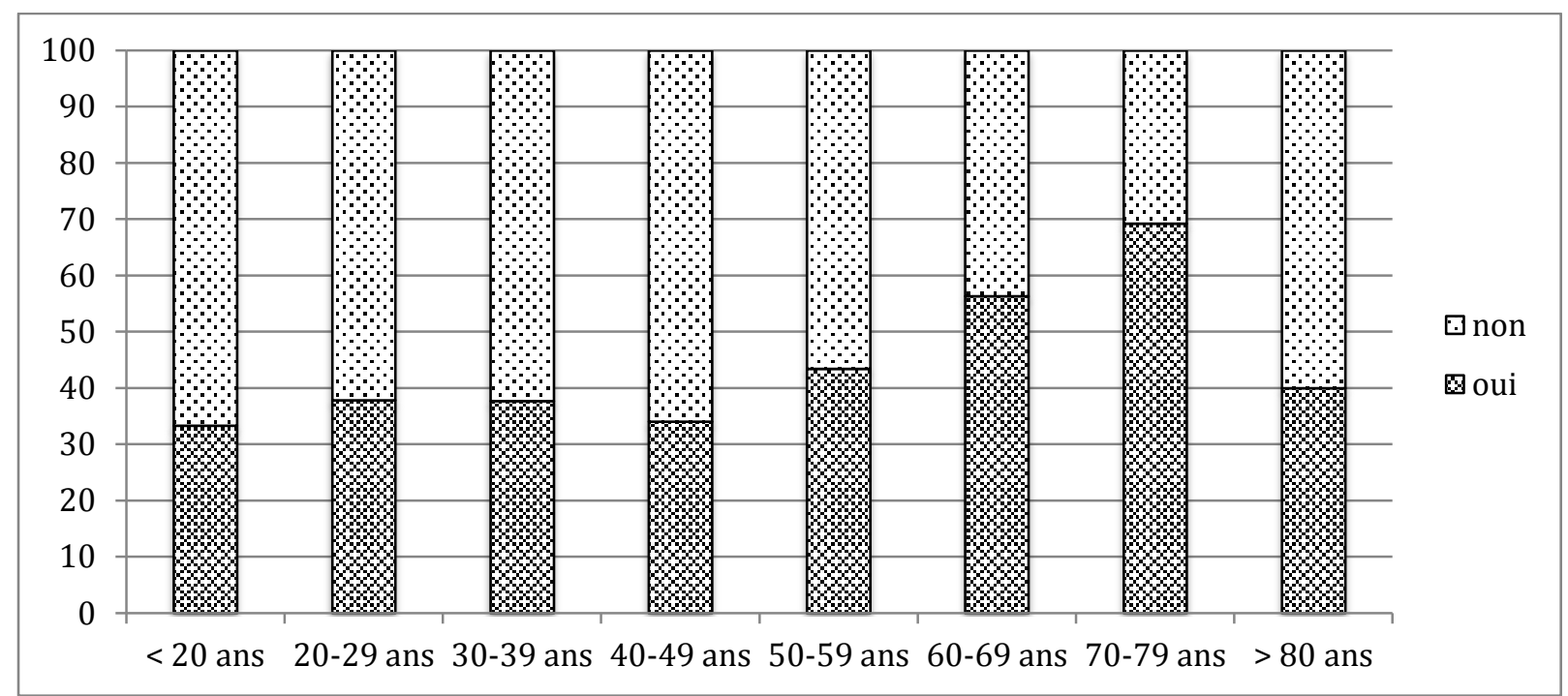

Figure 11. Identité vosgienne et tranche d'âge (\%) - «J'ai le sentiment d'être vosgien et ce sentiment est renforcé par l'écoute de VTV ».

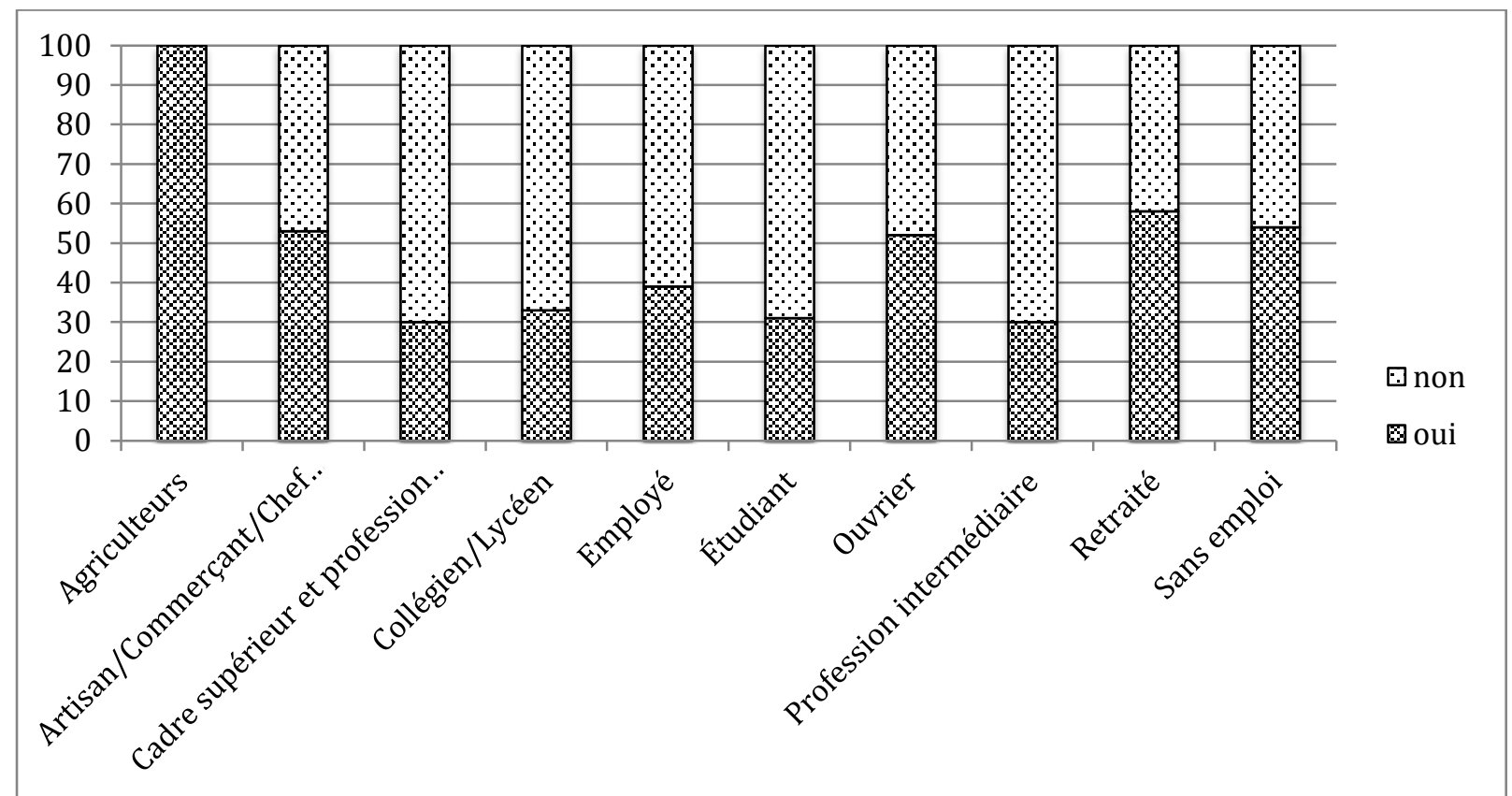

Figure 12. Identité territoriale et CSP (\%) - "J'ai le sentiment d'être vosgien et ce sentiment est renforcé par l'écoute de VTV ».

\section{Conclusion}

La réception de Vosges Télévision par ses téléspectateurs est plurielle mais plutôt consensuelle: la chaine est perçue comme informative et représentative des Vosges pour une majorité ; elle est espérée proche, sympathique, accessible et culturelle. Les personnes que nous avons rencontrées tendent à se présenter comme des téléspectateurs raisonnables et responsables, par opposition à l'image habituellement véhiculée d'une pratique télévisuelle passive, addictive et abêtissante - qu'ils contribuent eux-mêmes à diffuser. Ils attendent de leur chaîne locale la diffusion de programmes qui leur permettent de tenir cette posture : des programmes qui attisent leur curiosité, les cultivent; des programmes qui ne soient pas une simple source de 
distraction mais qui donnent envie d'être sélectionnés dans le cadre d'une écoute occasionnelle, en replay, qui se développe de plus en plus. Et ils attendent de Vosges Télévision d'être un acteur de premier plan de la vie locale.

Plus généralement, les résultats de l'enquête apportent un éclairage sur la relation des publics à la télévision a au moins deux échelles. D'une part, ils confirment l'idée selon laquelle la réception de la télévision se construit à travers les discours que les individus sont amenés à produire sur le média. Ici, on peut se demander, à l'instar de la réflexion proposée par Christine Servais (2018), si l'opinion formulée par les individus lorsqu'ils parlent de leur pratique télévisuelle est une opinion «qui se forge en vue d'autrui » 16 , c'est-à-dire en situation de communication projetée avec les autres, " en contexte», ce qui signifie que «toute réception est aussi une adresse à autrui ». En ce sens, la mise en récit de la pratique télévisuelle s'accompagne d'un sentiment de partage d'une opinion avec les autres qui participe de la formation d'un collectif, d'un « devenir public ». Nous avons observé cela au cours des trois séances de discussions de groupe, à travers un usage croissant du « on » dans les propos, non pour désigner une indétermination mais bien pour se référer au groupe de téléspectateurs ; il y a aussi les petites phrases comme "C'est ça que je voulais dire », ou encore une participation active à la conversation pour ceux qui se déclaraient en début de séance «non-téléspectateur».

D'autre part, nous remarquons combien le paradigme du local et de la proximité au sujet des télévisions locales est incorporé et revendiqué par les téléspectateurs, alors même que le visionnage des programmes de télévision locale pourrait s'accroitre grâce aux dispositifs socionumériques mis en place initialement pour donner accès à des programmes médiatiques d'un horizon plus large. La notion de service public se retrouve diluée au sein d'un ensemble d'attentes placées sous le signe d'une participation située à une échelle territoriale bornée. En ce sens, l'imaginaire de télévision locale produit dans les conversations combine le modèle d'une paléotélévision "fenêtre sur le monde » (s'informer, découvrir) à celui d'une télévision de l'intimité (Mehl, 1996) où doit être mise à l'écran et à l'honneur la parole de personnes ordinaires.

\section{Références}

BoulliER Dominique (1987), Les conversations télé, Rennes, Lares.

Boullier Dominique (2014), «La fabrique de l'opinion publique dans les conversations télé ». Réseaux, n¹26, p. 57-87.

BOURE Robert, LEFEBVRE Alain (2000). «Télévisions locales et territoires en mouvement. Vers un programme de recherches ». Hermès, n²6-27, p. 263-282.

Duchene Sophie, HAEgel Florence (2008), L'entretien collectif : l'enquête et ses méthodes, Paris, A. Colin.

GAdras Simon, PAILlart Isabelle (2013), «Les territoires et les médias dans la construction de l'espace public », in NOYER Jacques, PAILlart Isabelle, RAOUL Bruno, dirs,

\footnotetext{
${ }^{16}$ Par opposition à l'opinion reçue, c'est-à-dire le jugement déterminant qui circule dans l'espace public.
} 
Médias et territoire. L'espace public entre communication et imaginaire territorial, Lille, Presses universitaires du Septentrion, p. 23-38.

IDELSON Bernard (2013), «Les services publics de radio-télévision dans les DOM à l'ère du numérique: continuité des discours enchanteurs à propose de la "proximité", du "local" et de "l'ouverture sur le monde" ", Les enjeux de l'information et de la communication, $\mathrm{n}^{\circ} 14 / 2$, p. 135-148.

JEnKInS Henry (2013), La culture de la convergence. Des médias au transmédia, trad. de l'anglais par C. JAQUET, Paris, A. Colin/Ina éditions.

Jost François, dir. (2014), Pour une télévision de qualité, Paris, Ina éditions.

LAFON Benoit (2012), Histoire de la télévision régionale de la RTF à la 3, 1950-2012, Paris, Ina éditions.

Le Champion Rémy (2018), La télévision, Paris, Éd. La Découverte.

LE Guern Philippe, Leroux Pierre (2000), «Les limites de l'espace public médiatisé : l'exemple d'une télévision locale », Hermès, n²6-27, p. 159-173.

LEFEBURE Pierre (2011), «Les apports des entretiens collectifs à l'analyse des raisonnements politiques. Composition des groupes et dynamiques discursives », Revue française de science politique, $\mathrm{n}^{\circ} 61, \mathrm{p} .399-420$.

LEVY Marie-Françoise (2007), «Télévision, publics, citoyenneté. 1950-1974 », in CoHEN Évelyne, LEvy Marie-Françoise, dirs, La télévision des Trente glorieuses, culture et politique, Paris, CNRS éditions. p. 91-111.

MAIGRET Éric (2000), "La démocratie locale entre idéologies, identités et pratiques », Hermès, 26-27, p. 99-108.

MeAdel Cécile (2010), Quantifier le public. Histoire des mesures d'audience de la radio et de la télévision, Paris, Economica.

MeHL Dominique (1996), La télévision de l'intimité, Paris, Le Seuil.

PoEls Géraldine (2015), Les trente glorieuses du téléspectateur, Paris, Ina éditions.

SEguR Céline (2010), Les recherches sur les téléspectateurs. Trajectoire(s) académique(s), Paris, Hermès Lavoisier.

SERVAIS Christine (2018), "Adresse, opinion et responsabilité », in BALLARINI Loïc, SEgUR Céline, dirs, Devenir public. Modalités et enjeux, Paris, Éd. Mare \& Martin, p. 39-58. 